\title{
Trajectory Optimization of Electric Aircraft Subject to Subsystem Thermal Constraints
}

\author{
Robert D. Falck, ${ }^{*}$ Jeffrey C. Chin, ${ }^{\dagger}$ Sydney L. Schnulo, ${ }^{\ddagger}$ Jonathan M. Burt, ${ }^{\S}$ \\ and Justin S. Gray 9 \\ NASA Glenn Research Center, Cleveland, OH, 44135, U.S.A.
}

\begin{abstract}
Electric aircraft pose a unique design challenge in that they lack a simple way to reject waste heat from the power train. While conventional aircraft reject most of their excess heat in the exhaust stream, for electric aircraft this is not an option. To examine the implications of this challenge on electric aircraft design and performance, we developed a model of the electric subsystems for the NASA X-57 electric testbed aircraft. We then coupled this model with a model of simple 2D aircraft dynamics and used a Legendre-GaussLobatto collocation optimal control approach to find optimal trajectories for the aircraft with and without thermal constraints. The results show that the X-57 heat rejection systems are well designed for maximum-range and maximum-efficiency flight, without the need to deviate from an optimal trajectory. Stressing the thermal constraints by reducing the cooling capacity or requiring faster flight has a minimal impact on performance, as the trajectory optimization technique is able to find flight paths which honor the thermal constraints with relatively minor deviations from the nominal optimal trajectory.
\end{abstract}

\section{Nomenclature}

$\begin{array}{llll}A & \text { convective area } & p_{a t m} & \text { ambient atmospheric pressure } \\ C & \text { capacitance } & P & \text { power } \\ C_{D} & \text { drag coefficient } & \dot{Q} & \text { heat rate } \\ C_{h} & \text { heat capacity } & \mathbb{Q}_{m a x} & \text { battery cell maximum capacity } \\ C_{L} & \text { lift coefficient } & q & \text { dynamic pressure } \\ \mathcal{C}_{p} & \text { propeller power coefficient } & R & \text { resistance } \\ C_{p} & \text { specific heat at constant pressure } & \mathcal{R} & \text { residual } \\ C_{T} & \text { thrust coefficient } & r & \text { range } \\ D & \text { drag } & S & \text { battery state of charge } \\ d_{\text {prop }} & \text { propeller diameter } & t & \text { time } \\ F_{T} & \text { thrust } & T & \text { temperature } \\ \tilde{F}_{T} & \text { thrust required for steady flight } & U & \text { voltage } \\ h & \text { altitude } & v_{a} & \text { airspeed } \\ h_{c} & \text { convective heat transfer coefficient } & W & \text { total aircraft weight } \\ I & \text { current } & \alpha & \text { angle of attack } \\ J_{\text {prop }} & \text { propeller advance ratio } & \gamma & \text { flight path angle } \\ \ell_{w i r e} & \text { wire length per wing } & \gamma_{a} & \text { atmospheric ratio of specific heats } \\ L & \text { lift } & \eta & \text { efficiency } \\ \tilde{L} & \text { lift required for steady flight } & \rho_{a t m} & \text { atmospheric density } \\ \dot{m} & \text { mass flow rate } & \tau_{s h a f t} & \text { propeller shaft torque } \\ N & \text { propeller shaft revolutions per minute } & & \end{array}$

*Mission Architecture and Analysis Branch, rfalck@nasa.gov, AIAA Member.

${ }^{\dagger}$ Propulsion System Analysis Branch, jeffrey.c.chin@nasa.gov, AIAA Member.

¥Propulsion System Analysis Branch, sydney.l.schnulo@nasa.gov, AIAA Member.

$\S$ Mission Architecture and Analysis Branch, jonathan.m.burt@nasa.gov, AIAA Associate Fellow.

ฯPropulsion System Analysis Branch, justin.s.gray@nasa.gov, AIAA Member. 


\section{Introduction}

The study of electric aircraft for commercial use has grown in popularity in the last several years with a ride range of aircraft sizes and required missions being considered. ${ }^{1,2}$ NASA's X-57 "Maxwell" aircraft, developed under the Scalable Convergent Electric Propulsion Technology and Operations Research (SCEPTOR) program, ${ }^{3}$ is a twin propeller aircraft that serves as a proof-of-concept and test bed for a wide set of electric propulsion technologies including batteries, motors, and distributed electric propulsion. The X-57 features two main cruise motors on the wingtips with ten smaller electric motors and propellers for use in high-lift situations. For the purpose of this work, we only consider operation of the cruise motors.

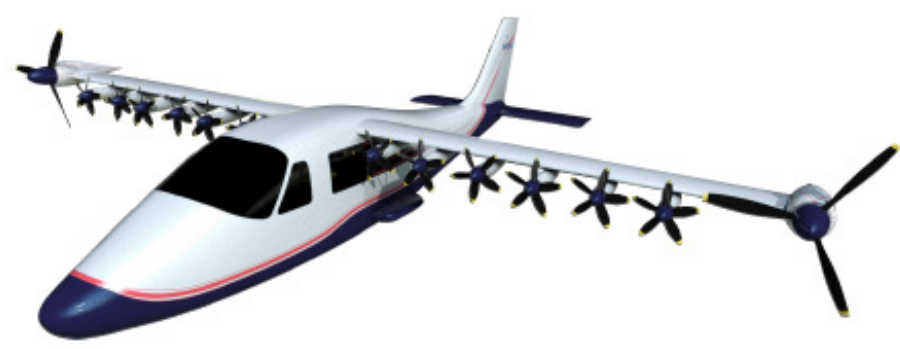

Figure 1. NASA's X-57 "Maxwell" aircraft

The development of X-57 has highlighted a unique challenge posed by the use of electric propulsion systems. Unlike piston engines or gas turbine based systems, for electric aircraft propulsion systems thermal management is a primary concern during the design process. While traditional propulsion systems are only around $60 \%$ thermally efficient, they produce waste heat in the form of exhaust air that is easily removed from the aircraft. In contrast, an electric propulsion system could be close to $90 \%$ efficient, but its waste heat is much more challenging to expel because there is no exhaust air with which to carry it. The motor, inverter, wires, and battery each generate heat that must be rejected to the atmosphere or else they will heat up to unacceptable temperatures over the course of a flight. Furthermore, these various components are expected to be located close to temperature sensitive power electronics and composite structures which must also be kept within acceptable temperature limits.

The amount of heat that needs to be rejected, and consequently the size of the required heat exchangers, is a function of the efficiency of the electrical components and their overall power output. Moreover, the power output of the propulsion system is not constant over the entire flight, hence the temperature profile of the various components is also not constant. It follows that the aircraft thermal management requirements can be posed as set of temperature limits that must be adhered to across the mission.

In this work we show that it is possible to partially mitigate the negative effects of these temperature limits by optimizing electric aircraft trajectories using a coupled propulsive-thermal-aerodynamic model. To demonstrate this we examine a series of trajectories for the X-57 aircraft subject to temperature constraints. We compare thermally constrained optimal trajectories against unconstrained optimal trajectories, and show that thermal constraints do alter the shape of the trajectory and can have a meaningful impact on overall performance.

Although considerable bodies of work exist on trajectory analysis for electric aircraft and on thermally constrained aircraft propulsion system analysis, few previous studies have considered the two issues simultaneously. Doman considered optimal cruise altitudes for aircraft subject to fuel tank temperature constraints. ${ }^{4}$ Alyanak and Allison showed that thermal considerations for military aircraft could lead to a significant increase in takeoff gross weight if not properly accounted for in the early design process. ${ }^{5}$ Vegh, Alonso, Tarik, and de Silva demonstrated combined flight path and aircraft design optimization for electric passenger aircraft, although their model did not include thermal constraints due to waste heat of the propulsion system. ${ }^{6}$ Antcliff, Guynn, et al. demonstrated combined mission analysis and sizing of a hybrid electric aircraft in a multi-disciplinary optimization environment without explicit consideration of thermal constraints. ${ }^{7}$ Christie studied the effectiveness of various cooling methods for removing the excess heat generated by electric propulsion components, concluding that heat-exchanger surface area was one of the most critical factors in determined cooling effectiveness. ${ }^{8}$ Dubois showed that increasing heat exchanger area also increases air- 
craft drag. ${ }^{9}$ Although neither Christie's nor Dubois's work directly considered trajectory analysis, together they establish competing trends which indicate that heat rejection from electric propulsion components is a significant design challenge.

The model used in this work integrates thermal analysis of the electric motor, batteries, inverter, and power transmission wiring with a trajectory analysis model to simultaneously capture the the effects of both. Trajectories are found using an optimal control approach based on a Legendre-Gauss-Lobatto (LGL) collocation method with gradient based optimization and analytic derivatives. This method, developed by Herman and Conway, ${ }^{10}$ is a generalization of Hermite-Simpson transcription of Paris and Hargraves. ${ }^{11}$ We performed trade studies on this model to characterize the effect of the thermal constraints on the maximumrange, maximum-efficiency, and minimum-time trajectories of an electrically powered aircraft modeled after NASA's experimental X-57.

The results of the studies performed in this work show that thermal constraints, especially on the motors, can strongly limit the achievable climb rate. If a particular climb rate capability is required, then sufficient cooling may need to be incorporated into the aircraft design. Alternatively, our analysis suggests that aircraft can handle insufficient cooling via modest reductions in the climb rate without significantly reducing the maximum range. Aircraft range is primarily restricted by the energy capacity of the batteries, and a modest thermally-unconstrained optimal climb profile will have only a small impact on net energy usage.

\section{Approach}

We utilize an LGL collocation scheme built upon the OpenMDAO framework, ${ }^{12}$ as previously demonstrated by Falck, Gray, and Naylor ${ }^{13}$ for aircraft trajectory optimization. In addition to collocating position and velocity state variables, we assign the temperatures of electrical components, the battery state of charge, and the Thevenin voltage of the battery model as collocated states. Derivatives for the optimizer and the Newton solvers are provided analytically. OpenMDAO uses the unified derivative approach developed by Martins and Hwang ${ }^{14}$ to provide analytic derivatives despite the presence of several iterative solvers in the model.

\section{A. Aircraft 2D Equations of Motion}

For this work a simplified set of equations of motion for an aircraft in a 2D rectilinear gravity field is assumed. Furthermore, an assumption of steady flight is imposed. This approach, developed by Kao, Hwang, Martins, Gray, and Moore, ${ }^{15}$ simplifies the collocation by removing potentially oscillatory behavior in the states and controls.

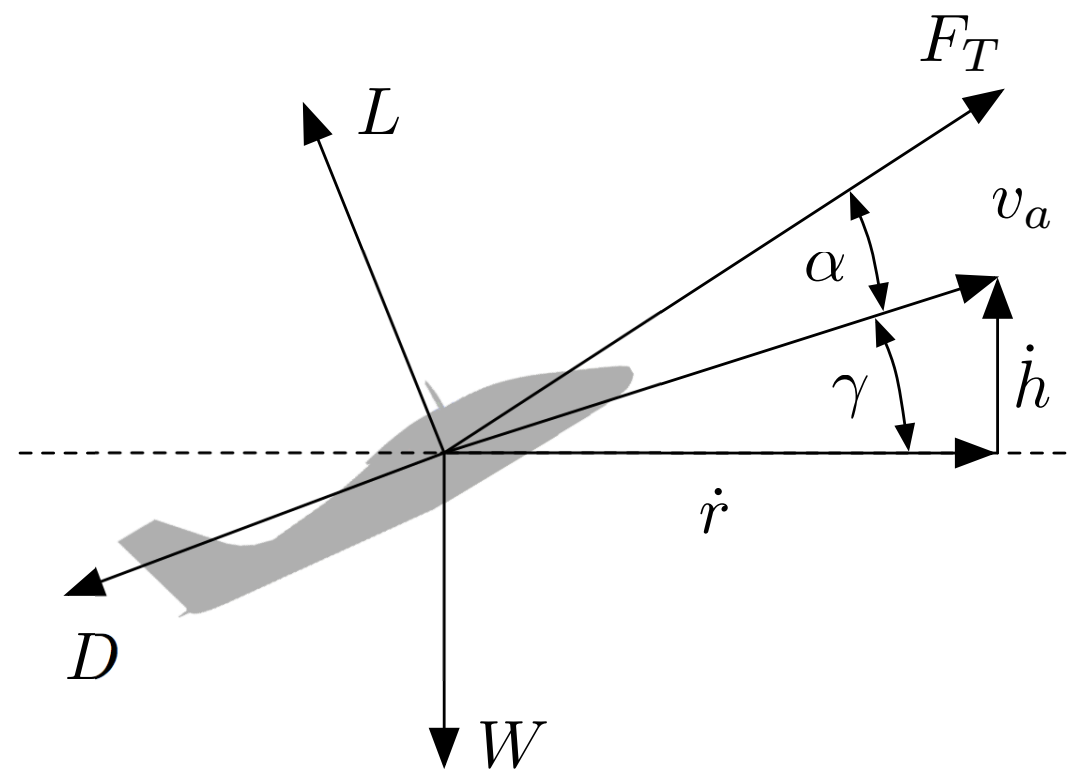

Figure 2. Free-body diagram for the derivation of the steady flight conditions. 
In this approach, range is a state variable to be implicitly integrated, whereas altitude and airspeed are dynamic controls. Since the aircraft expends no fuel we treat aircraft weight as a parameter rather than a state variable. Assuming a wind-free atmosphere, the equation of motion governing range is:

$$
\dot{r}=v_{a} \cos \gamma
$$

The aircraft altitude and airspeed time-histories are prescribed at the control nodes of the collocation method. We apply the steady flight assumption by neglecting any acceleration in the vehicle force balance. From the free-body diagram in Figure 2, if we assume zero acceleration in the aerodynamic axes we have:

$$
\begin{aligned}
& 0=F_{T} \cos \alpha-W \sin \gamma-D \\
& 0=L-W \cos \gamma+F_{T} \sin \alpha
\end{aligned}
$$

Rearranging (2) and (3) provides the values of lift and thrust required to satisfy the steady flight condition.

$$
\begin{aligned}
\tilde{L} & =W \cos \gamma-F_{T} \sin \alpha \\
\tilde{F}_{T} & =\frac{W \sin \gamma}{\cos \alpha}+\frac{D}{\cos \alpha}
\end{aligned}
$$

A Newton solver is used to solve for the values of $\alpha$ and $P_{i n-m o t o r}$ such that the computed values of lift and thrust equal those required for steady flight. The residual equations for each are:

$$
\begin{aligned}
\mathcal{R}(\alpha) & =L(\alpha)-\tilde{L} \\
\mathcal{R}\left(P_{\text {in-motor }}\right) & =F_{T}\left(P_{\text {in-motor }}\right)-\tilde{F}_{T}
\end{aligned}
$$

The lift coefficient is based on a simple quadratic fit to the angle of attack $(\alpha)$, where $\alpha$ is in radians.

$$
C_{L}=-3.8500 \alpha^{2}+4.8923 \alpha+0.6865
$$

The drag coefficient is interpolated as a function of airspeed and the lift coefficient.

$$
C_{D}=f\left(C_{L}, v_{a}\right)
$$

Since the collocation method uses vehicle altitude as a dynamic control variable, we can use the control differentiation matrix $\left(\left[D_{u}\right]\right)$ from the collocation formulation to obtain an approximate time-derivative of altitude.

$$
\dot{h}=\frac{2\left[D_{u}\right] h}{t_{\text {seg }}}
$$

The flight path angle can then be computed based on the values of the true airspeed and the vertical velocity component:

$$
\gamma=\arcsin \frac{\dot{h}}{v_{a}}
$$

$$
4 \text { of } 16
$$




\section{B. Propeller Model}

The propeller model is responsible for providing the thrust force to the equations of motion. Thrust generated by the propellers is computed as

$$
F_{T}=2 C_{T} \rho\left(\frac{N}{60}\right)^{2} d_{\text {prop }}^{4}
$$

where $\rho$ is the ambient atmospheric density, $\mathrm{N}$ is the propeller speed in revolutions per minute, and $d_{\text {prop }}$ is the propeller diameter. The factor of two is included to simplify the analysis by assuming identical operation in each of the aircraft's two cruise motors/propellers. The coefficient of thrust $C_{T}$ is

$$
C_{T}=\frac{\mathcal{C}_{p} \eta_{\text {prop }}}{J_{\text {prop }}}
$$

where $\mathcal{C}_{p}$ is the coefficient of power of the propeller, computed as:

$$
\mathcal{C}_{p}=\frac{P_{\text {shaft }}}{\rho\left(\frac{N}{60}\right)^{3} d_{\text {prop }}^{5}}
$$

The propeller efficiency $\eta_{\text {prop }}$ is interpolated as a function of shaft torque $\tau_{\text {shaft }}$ and the advance ratio $J_{T}$ :

$$
\eta_{\text {prop }}=f\left(\tau_{\text {shaft }}, J_{\text {prop }}\right)
$$

where the advance ratio is

$$
J_{\text {prop }}=\frac{60 v_{a}}{N d_{\text {prop }}}
$$

and shaft torque is given in $\mathrm{Nm}$ as

$$
\tau_{\text {shaft }}=\frac{60 P_{\text {shaft }}}{2 \pi N}
$$

Performance maps for Equation 15 are provided by the propeller manufacturer, and are interpolated using a B-spline interpolant.

\section{Electrical Component Models}

For this study the electrical components are modeled as a single string of components from battery pack through motor. We assume both motors and propellers have the same inputs and simply double the resulting thrust before sending it to the equations of motion. The extended design structure matrix of the electrical subsystems is shown in Figure $3 .{ }^{16}$ Since we use motor input power as a control variable, we include battery output power as an implicit state variable to be converged by a Newton solver. Outputs from the electrical subsystem are the temperature time derivatives of the components (battery, wire, inverter, and motor), as well as the time derivatives for the state-of-charge and the Thevenin voltage of the battery. These are used in the implicit integration of their corresponding states in the optimal control problem. 


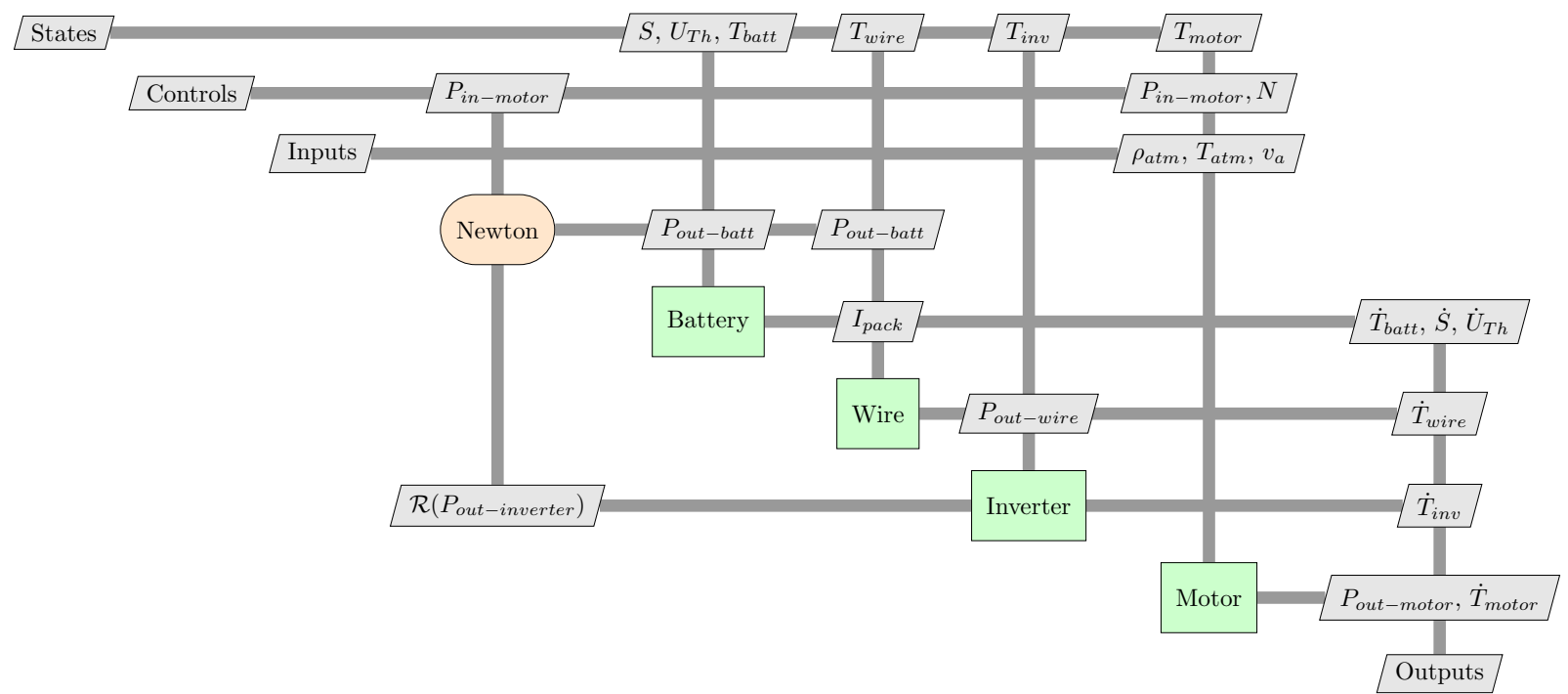

Figure 3. The Extended Design Structure Matrix of the electrical subsystems models.

\section{Transient Battery Model}

In order to capture thermal effects within the battery and subsequent components, a model is required to simulate battery internal resistance and voltage as a function of current draw, state of charge (S), and battery temperature. Existing models for Lithium Ion batteries are explored in detail in multiple studies. ${ }^{17,18}$ These models are composed of equivalent circuit models of varying complexity including. The Thevenin model was identified as the ideal model for this application due to its simplicity and data availability, while still being able to capture transient effects and track state of charge within $2 \%$ accuracy of experimental data. This model - shown in Figure 4- is composed of a voltage source, an internal resistance, and a parallel RC block to capture polarization effects.

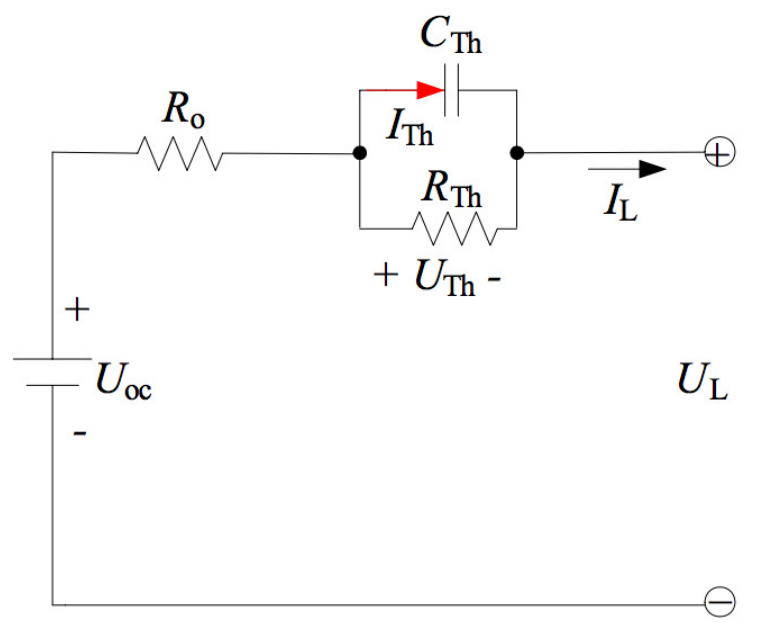

Figure 4. Thevenin equivalent circuit model of a battery containing a transient RC block.

The values of each of these circuit components are interpolated from performance maps as a function of the state of charge and current temperature,

$$
U_{o c}, C_{T h}, R_{0}, R_{T h}=f\left(S, T_{b a t t}\right)
$$


Subscripts $o c, T h$ and $L$ in Figure 4 and Equation 18 refer to open circuit, Thevenin and line, respectively. The Thevenin voltage $U_{T h}$, battery state of charge $S$ and battery temperature $T_{\text {batt }}$ are treated as integrated states over the course of the simulation, subject to the following differential equations: ${ }^{18}$

$$
\begin{aligned}
\dot{U}_{T h} & =\frac{-U_{T h}}{R_{T h} C_{T h}}+\frac{I_{L}}{C_{T h}} \\
\dot{S} & =\frac{-I_{L}}{\mathbb{Q}_{\text {max }}} \\
\dot{T}_{\text {batt }} & =\left(1-\eta_{\text {cell }}\right) \frac{I_{L} U_{L}}{m_{\text {cell }} C p_{\text {cell }}}
\end{aligned}
$$

Where $\mathbb{Q}_{\max }$ is the capacity of a single cell, which is $3 A h$ in our model. Each of the aircraft's two battery packs are arranged with 128 cells in series $\left(n_{\text {series }}\right)$, and 40 in parallel $\left(n_{\text {parallel }}\right)$. The line voltage is computed from the open circuit and Thevenin voltage as:

$$
U_{L}=U_{o c}-U_{T h}-I_{L} R_{0}
$$

The electric properties of the battery pack are then:

$$
\begin{aligned}
I_{\text {pack }} & =I_{L} \cdot n_{\text {parallel }} \\
U_{\text {pack }} & =U_{L} \cdot n_{\text {series }} \\
P_{\text {pack }} & =I_{\text {pack }} \cdot U_{\text {pack }}
\end{aligned}
$$

In order to satisfy the equations of motion for the aircraft a demanded power can be computed. A Newton solver is then used to find the current load on a single cell $\left(I_{L}\right)$ such that the power output back the battery pack is equal to the demanded power:

$$
\mathcal{R}\left(I_{L}\right)=P_{\text {out-battery }}-P_{\text {pack }}
$$

The battery output power is determined by reducing the requested motor input power by the efficiencies of the wire and inverter. Using this model, the heat output of the battery and voltage of the batteries can be accurately tracked. The battery model also dictates the current and voltage levels supplied to the other electric components, which is critical for estimating their thermal loads.

\section{High Power Wire Model}

Tracking heat accumulation across the long cable runs within the wings is necessary due to the stringent temperature constraints associated with the composite resins used to construct the structural ducting. Heat lost by the power bus results in Joule heating. In order to calculate this, the current through the power bus is divided by the number of wires that reside. The heat transfer rate is then calculated by the following equation:

$$
\begin{aligned}
& \dot{Q}_{\text {wire }}=I_{\text {wire }}^{2} \cdot R_{\text {wire }} \\
& \dot{Q}_{\text {cool-wire }}=c_{\text {ins }}\left(T_{\text {wire }}-T_{\text {amb }}\right) h_{c, \text { wire }} \ell_{\text {wire }} \\
& \dot{T}_{\text {wire }}=\left(\dot{Q}_{\text {wire }}-\dot{Q}_{\text {cool-wire }}\right) C_{h, \text { wire }} / \ell_{\text {wire }} \\
& 7 \text { of } 16
\end{aligned}
$$


where $c_{i n s}$ is the circumference of the wire insulation jacket, $h_{c, w i r e}$ is the overall convective heat transfer coefficient of the wiring, and $C_{h \text {,wire }}$ is the heat capacity of the wire. The power bus is considered under natural convection at NASA Armstrong Flight Research Center "hot day" temperatures $\left(T_{a m b}\right)$. Because the wing housing the power buses allows only minimal airflow, this assumption provides for a conservative estimate of the temperature within the wing.

\section{Motor Model}

The motor component determines motor efficiency based on RPM and torque demanded by the propeller. This efficiency is computed using an interpolated performance map provided by the motor manufacturer.

Motor efficiency is used to determine the power loss across the motor component, which is converted to heat. The cruise motors are passively cooled by allowing air to pass through the cruise nacelle. In order to increase their heat transfer surface area, a finned heat sink is connected to the stator coils. After air passes through this heat sink, it is ducted to the inverters that are installed behind the motor in the cruise nacelle. This creates a circular relationship between the motor and the inverters in the component modeling; the inverters provide power to the motor and the motor rejects heat into the air-stream that will in turn cool the inverter. The following two equations calculate the rate of motor heat production $\left(\dot{Q}_{\text {motor }}\right)$ and heat dissipation $\left(\dot{Q}_{\text {cool-motor }}\right)$ :

$$
\begin{gathered}
\dot{Q}_{\text {motor }}=P_{\text {in-motor }}\left(1-\eta_{\text {motor }}\right) \\
\dot{Q}_{\text {cool-motor }}=h_{c, \text { motor }} \cdot A_{\text {motor }}\left(T_{\text {motor }}-T_{a t m}\right)
\end{gathered}
$$

where $P_{\text {in-motor }}$ is the motor input power and $T_{a t m}$ is the ambient atmospheric temperature. The difference between these two values yields a change in temperature within the motor. The rate at which temperature changes is found using the motor's heat capacity.

$$
\dot{T}_{\text {motor }}=\left(\dot{Q}_{\text {motor }}-\dot{Q}_{\text {cool-motor }}\right) / C_{h, \text { motor }}
$$

Because the inverters are being cooled by the motor exhaust, it is important to calculate the temperature of that exhaust air that will be used in the inverter component.

$$
T_{e x h}=T_{a t m}+\frac{\dot{Q}_{c o o l-m o t o r}}{\dot{m} \cdot C p_{a i r}}
$$

Here $T_{e x h}$ is the temperature of the exhausted cooling air and $\dot{m}$ is the mass flow rate of cooling air.

\section{Inverter Model}

Two inverters drive each cruise motor in the X-57 configuration. Because the inverters are not $100 \%$ efficient, they carry a thermal load that may constrain the mission performance. Each inverter is equipped with a finned heat sink to improve heat transfer from the inverter chip to the air being passed through the motor nacelle. The process to model inverter temperature mirrors that of the motor, except that the inverter is exposed to the motor exhaust rather than ambient air.

$$
\begin{aligned}
\dot{Q}_{i n v} & =P_{i n-i n v}\left(1-\eta_{i n v}\right) \\
\dot{Q}_{c o o l-i n v} & =h_{c, i n v} \cdot A_{i n v}\left(T_{i n v}-T_{e x h}\right)
\end{aligned}
$$

In the above equations, $\dot{Q}_{i n v}$ represents the heat generation rate for each inverter, and $\dot{Q}_{\text {cool-inv }}$ is the rate at which heat is being rejected to the air stream through the fins. The heat transfer coefficient, 
$h_{c, \text { inv }}$, represents the forced convection that the inverter experiences. To determine the rate of change of temperature, the net heat transfer is divided by the heat capacity, $C_{h, i n v}$, of the inverter.

$$
\dot{T}_{i n v}=\left(\dot{Q}_{i n v}-\dot{Q}_{c o o l-i n v}\right) / C_{h, i n v}
$$

\section{Optimal Control Method}

Using the dynamics above, the authors devised several optimal control problems. These optimal control problems were posed using an LGL-based approach built in the OpenMDAO environment previously described in reference 13. The optimal control problem is transcribed to a nonlinear programming problem which is subsequently solved with the SNOPT optimization software package. ${ }^{19}$

The state variables of the optimal control problem $(\vec{X})$ are the range, battery state of charge, battery Thevenin voltage, battery temperature, wire temperature, inverter temperature, and motor temperature:

$$
\vec{X}=\left[r, S, U_{T h}, T_{b a t t}, T_{w i r e}, T_{i n v}, T_{\text {motor }}\right]
$$

The dynamic control variables of the problem $(\vec{U})$ are altitude, airspeed, and motor/propeller shaft speed:

$$
\vec{U}=\left[h, v_{a}, N\right]
$$

Notably absent from the set of control variables are the angle-of-attack and motor power. Rather than letting the optimizer determine the angle of attack and motor input power, those quantities are determined by an internal Newton solver such that the steady-flight assumption is enforced.

For all calculations presented here, grids of 20 3rd-order segments or 11 th-order segments achieved good convergence with accurate representation of the state variables. Results were numerically integrated using SciPy to ensure reasonable accuracy of the state time histories compared to the collocated solution. ${ }^{20}$ Plots below show the results of numerical integration of the dynamics subject to initial state values, the optimal final time, and interpolated values of the optimal control profile.

\section{Analysis and results}

In the following sections we demonstrate some optimized trajectories of the electric aircraft model with and without thermal constraints imposed. First, we demonstrate cases which maximize the range covered. The second set of cases represent maximum efficiency flight to fly a given distance, analogous to a minimumfuel trajectory in a fuel-burning aircraft. Finally, we demonstrate minimum time trajectories which fly the desired range subject to a minimum allowable state of charge at the end of the trajectory.

The X-57 aircraft includes a novel distributed electric propulsion based high lift system which is not modeled in the present work. The trajectory is assumed to begin and end at an altitude of $1000 \mathrm{~m}$, where the distributed propulsion system is assumed to be deactivated. The initial and final airspeed and flight path angle are determined by the optimizer. The climb and descent rates are constrained to $3.81 \mathrm{~m} / \mathrm{s}(750 \mathrm{ft} / \mathrm{min})$, and the gross mass of the aircraft is $1174.8 \mathrm{~kg}(2590 \mathrm{lbs})$. As well will show, the optimization is somewhat insensitive to acceleration. To help reduce noise in the optimal velocity profile and aid convergence, the acceleration is limited to $\pm 0.025 \mathrm{~m} / \mathrm{s}$.

We begin the trajectories with a state of charge of 0.95 , attributing the first $5 \%$ to the climb to $1000 \mathrm{~m}$. Unless otherwise noted, we consider $40 \%$ to be the minimum allowable remaining state of charge at the end of the trajectory. This is provided as a margin to allow the aircraft enough remaining energy to loiter and land. We also impose a motor temperature limit of $100{ }^{\circ} \mathrm{C}$. The model tracks temperatures of multiple

components in the electrical system (battery, wiring, inverter, motor) but in all cases the motor temperature is the limiting factor. 


\section{A. Maximum Range Trajectory}

In this case, we examine the behavior of maximum range trajectories for electric aircraft. Results are shown in Figure 5. The optimal control problem examined here is:

$$
\begin{aligned}
\operatorname{maximize} \quad J=r\left(t_{f}\right) & \\
\text { subject to } \quad T_{\text {motor }}(t) & \leq 100^{\circ} \mathrm{C} \\
h\left(t_{0}\right) & =1000 \mathrm{~m} \\
h\left(t_{f}\right) & =1000 \mathrm{~m} \\
0.4 & \leq S(t) \leq 0.95 \\
-3.81 \mathrm{~m} / \mathrm{s} & \leq \dot{h}(t) \leq 3.81 \mathrm{~m} / \mathrm{s} \\
-0.025 \mathrm{~m} / \mathrm{s}^{2} & \leq \dot{v}_{a}(t) \leq 0.025 \mathrm{~m} / \mathrm{s}^{2}
\end{aligned}
$$
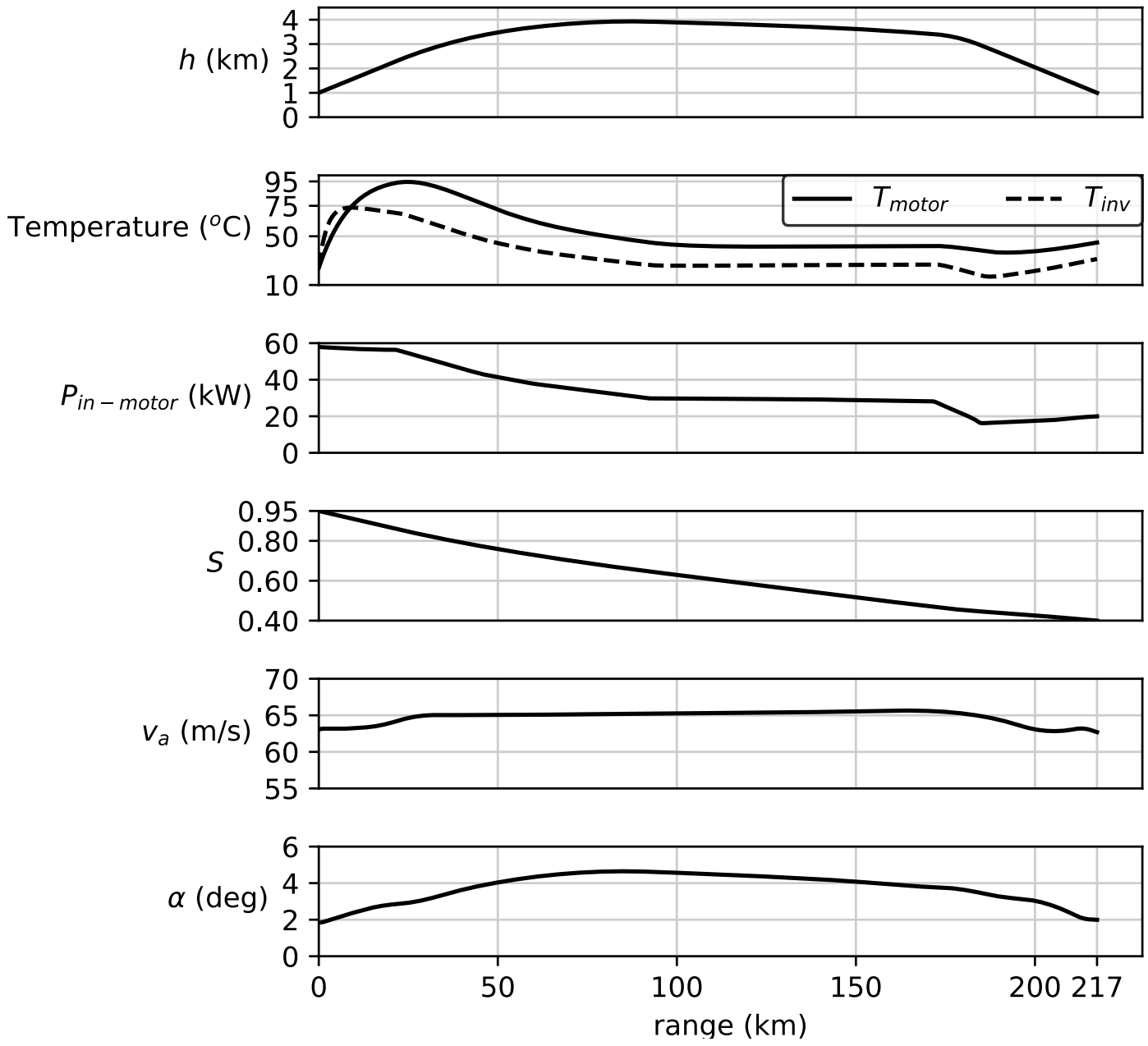

Figure 5. Key parameters of the maximum range trajectory. The aircraft reaches a range of $217 \mathrm{~km}$ with a cruise speed of about $65 \mathrm{~m} / \mathrm{s}$.

Notable trends in Figure 5 include a shallow descent over much of the cruise phase, and climb and final descent phases which both meet the maximum $|\dot{h}|$ constraint of $3.81 \mathrm{~m} / \mathrm{s}$ as indicated by near-constant 
values of the motor input power. An optimal airspeed of approximately $65 \mathrm{~m} / \mathrm{s}$ is maintained for nearly the entire trajectory. It is also shown in Figure 5 that the motor temperature does not exceed the $100{ }^{\circ} \mathrm{C}$ limit, indicating that the heat rejection systems on the X-57 are adequately sized for this problem.

Having demonstrated in Figure 5 that the heat rejection systems on the X-57 are adequately sized for our maximum range case, one might naturally ask what happens to the range performance if we significantly reduce the heat rejection area of the motor. To investigate this scenario, we consider a modified maximum range problem for which the area of the motor's heat rejection system is reduced by $30 \%$. The maximum range of the aircraft in that case reduced by by only $1.0 \mathrm{~km}$, from $217.3 \mathrm{~km}$ to $216.3 \mathrm{~km}$.
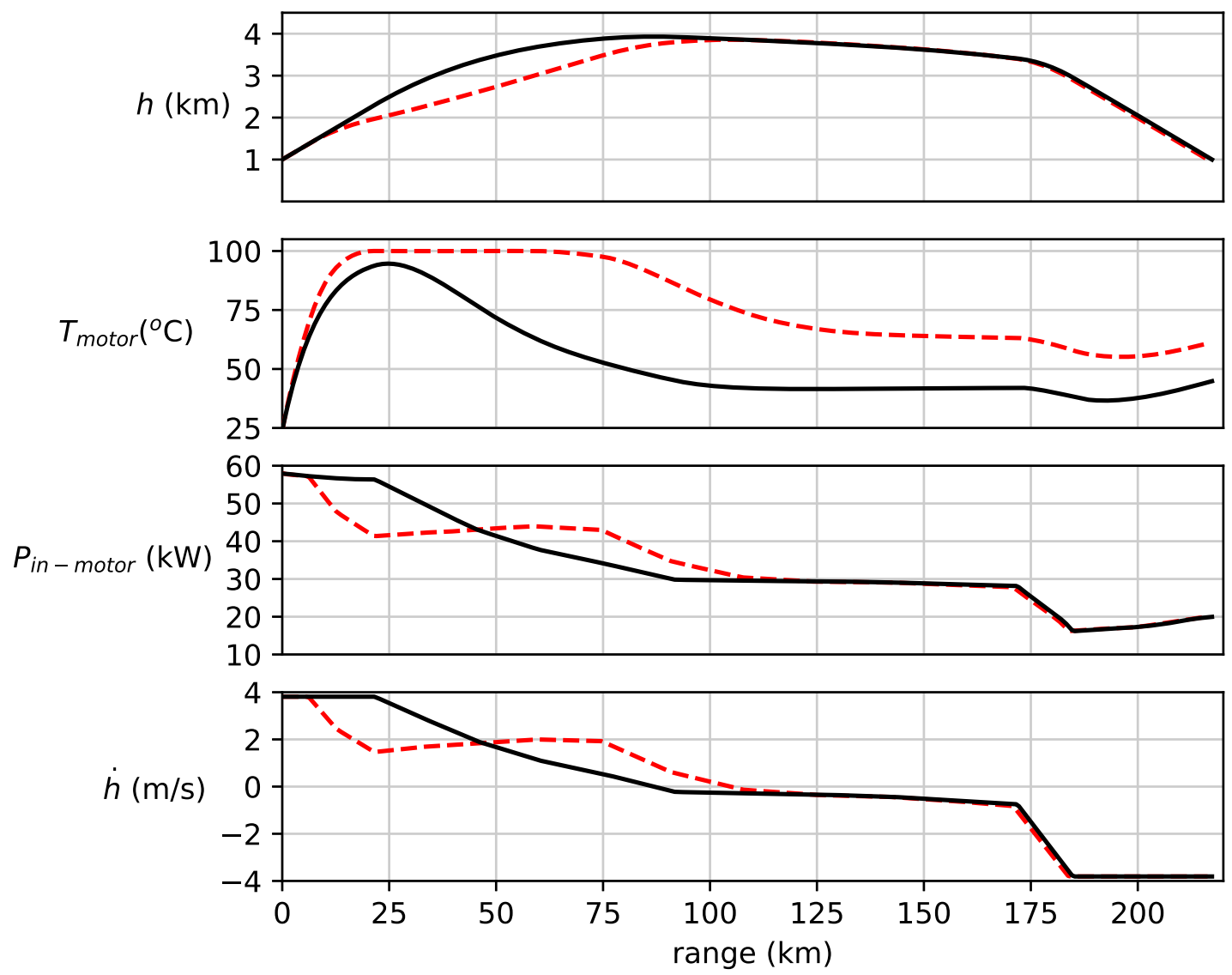

nominal heat exchanger area _ _-- reduced heat exchanger area

Figure 6. Reducing the motor heat exchanger area by $30 \%$ has little impact on the maximum range. The trajectory addresses the motor temperature limit by reducing rate of climb $(\dot{h})$.

In comparison to the nominal case, it is shown in Figure 6 that reducing the heat exchanger area causes the maximum range trajectory to exhibit a reduced climb rate. However, the trajectory with the reduced heat rejection capability maintains this reduced climb rate for a longer duration. In effect, after the initial climb rate reduction, the aircraft adds power to "catch up" to the nominal maximum range trajectory. The total energy spent is nearly the same hence the minimal reduction in the achievable range.

Notably, while thermal constraints do not strongly affect overall range, these constraints do significantly influence the rate-of-climb performance of the aircraft. Additionally, the maximum power output of the motors in the thermally constrained case (neglecting a very short duration at the beginning) is only about 45 kilowatts compared to 58 kilowatts for the unconstrained case. By reducing the heat exchanger area, and causing a thermal constraint, we have limited the power output of the motor. In theory if the smaller heat exchanger area was all that could be fit onto the airframe, The propulsion system could then be redesigned to the lower continuous power level and become lighter. 


\section{B. Maximum Efficiency Trajectory}

In this case we seek to maximize the final charge of the battery given some fixed range of flight.

$$
\begin{aligned}
& \operatorname{maximize} \quad J=S\left(t_{f}\right) \\
& \text { subject to } \quad r\left(t_{f}\right)=200 \mathrm{~km} \\
& T_{\text {motor }}(t) \leq 100{ }^{\circ} \mathrm{C} \\
& h\left(t_{0}\right)=1000 \mathrm{~m} \\
& h\left(t_{f}\right)=1000 m \\
& -3.81 \mathrm{~m} / \mathrm{s} \leq \dot{h}(t) \leq 3.81 \mathrm{~m} / \mathrm{s} \\
& -0.025 \mathrm{~m} / \mathrm{s}^{2} \leq \dot{v}_{a}(t) \leq 0.025 \mathrm{~m} / \mathrm{s}^{2}
\end{aligned}
$$
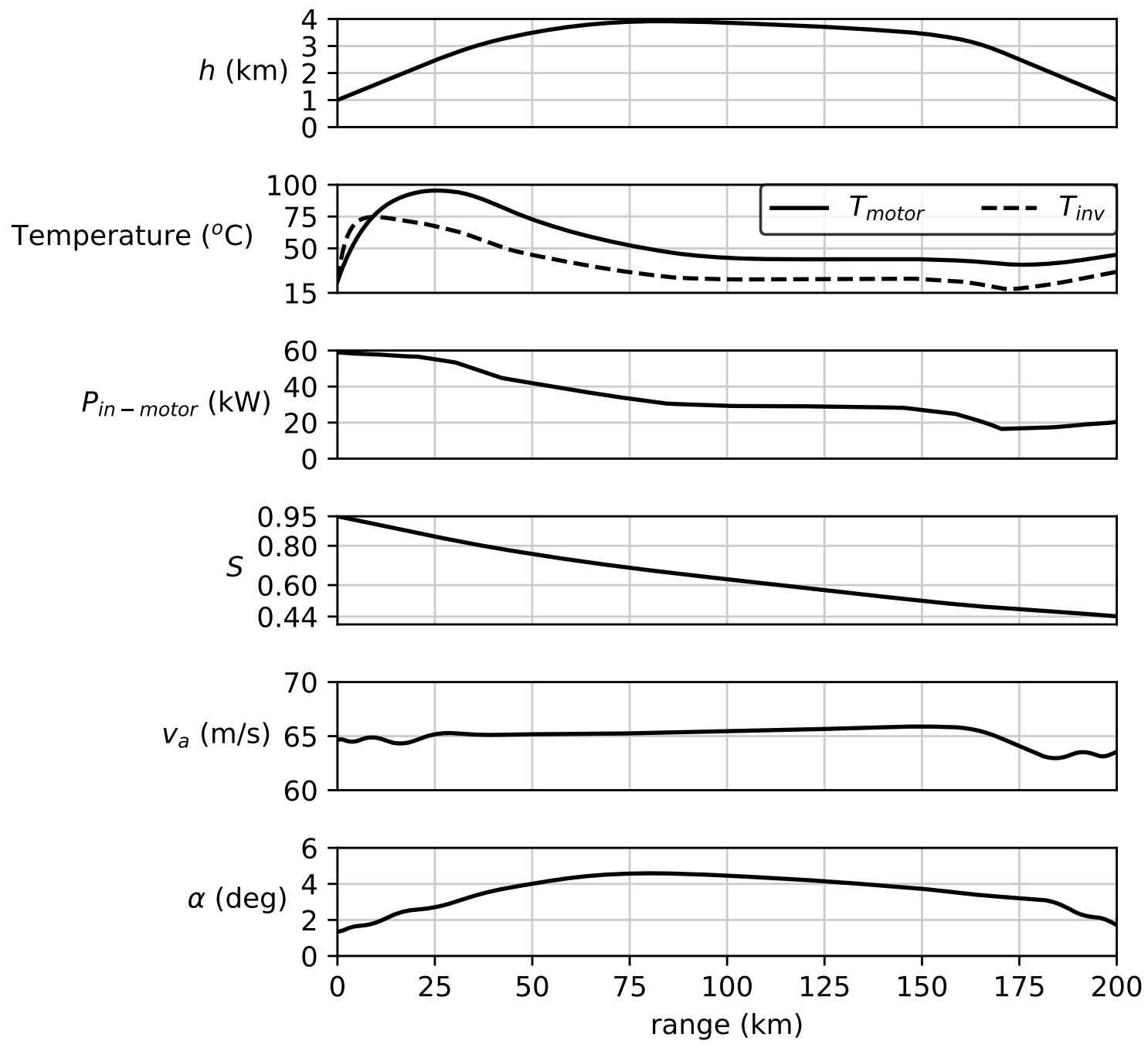

Figure 7. Results of the maximum efficiency trajectory. 
Key parameters of the resulting trajectory are shown in Figure 7. The aircraft flies $200 \mathrm{~km}$ in 51.3 minutes with a final state-of-charge of $44 \%$. The motor reaches a peak temperature of $95.4^{\circ} \mathrm{C}$, again demonstrating that the heat management systems of the vehicle are adequate. The aircraft flies at about the same cruising speed as the maximum range case, but the trajectory includes less altitude variation during cruise and a smaller cruise angle-of-attack to conserve energy.

\section{Minimum Time Trajectory}

In general, maximizing efficiency at the cost of increased flight duration may not be desirable. However, shorter flight times (higher average speeds) require more motor power and result in trajectories which violate the motor and inverter thermal constraints if these constraints are not explicitly enforced. By shaping the trajectory with thermal constraints in mind, it is possible to increase the average speed without violating the thermal constraints. In this case we modify the maximum efficiency problem by instead minimizing the final time $\left(t_{f}\right)$ and setting the minimal allowable state of charge to $40 \%$.
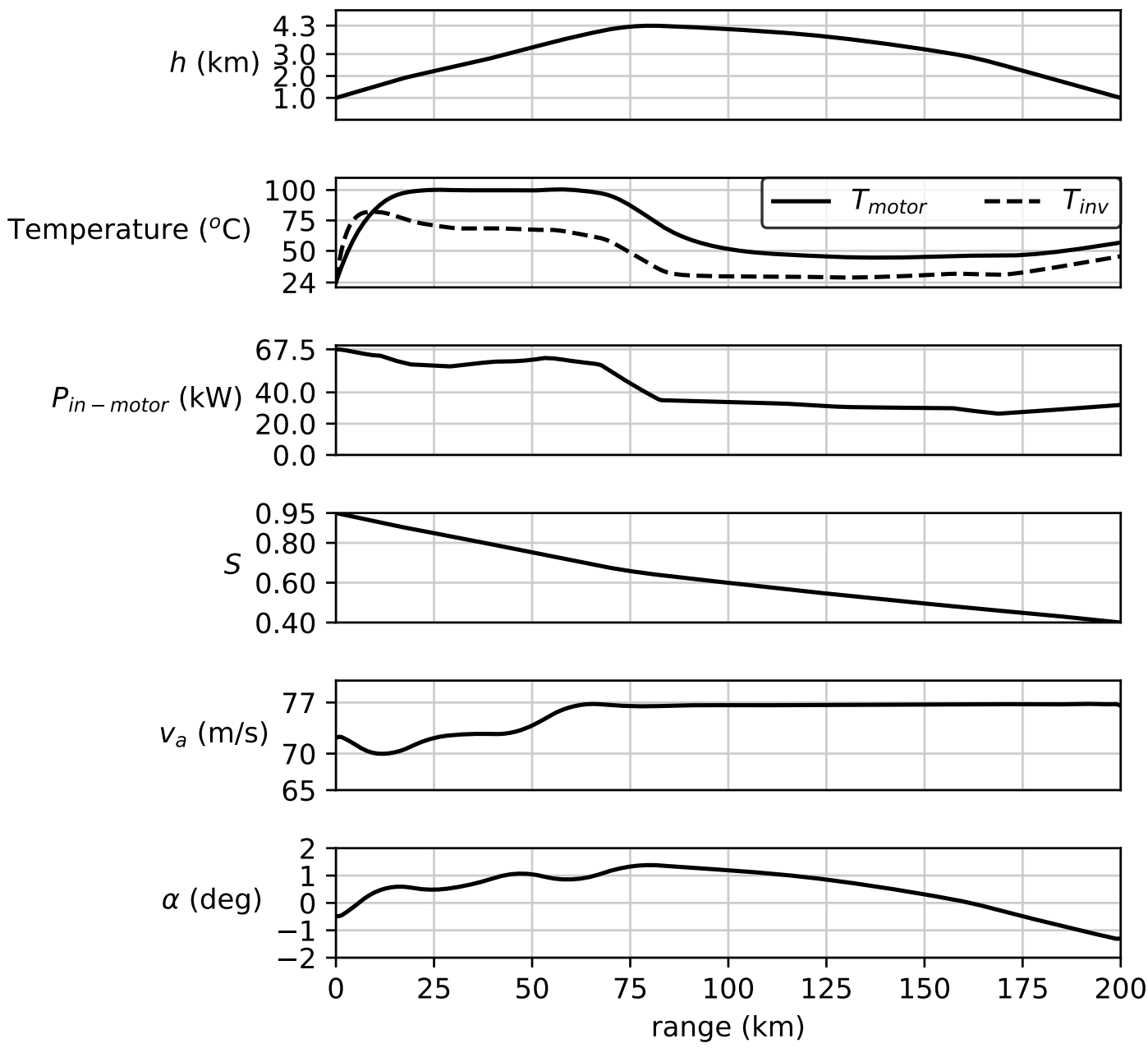

Figure 8. Key parameters for the minimum time plot with motor temperature constrained to $100{ }^{\circ} \mathrm{C}$.

As shown in Figure 8, upon reaching peak altitude the trajectory has a significantly higher airspeed than the maximum range and maximum efficiency cases. By modulating motor input power and climb rate to ride the thermal constraint boundary, we can modestly increase the average speed over a $200 \mathrm{~km}$ trajectory. The time of flight is roughly 44.23 minutes, compared to 51.3 minutes for the maximum efficiency case. Unlike the maximum range and maximum efficiency cases, the minimum time case demonstrates no cruise 
phase; instead the aircraft climbs to a maximum altitude of $4.3 \mathrm{~km}$ as quickly as possible, and then begins a continuous descent.

Running the minimum-time case without constraints on the motor temperature demonstrates that thermal constraints are far less constraining than the limit on the final state of charge. If the aircraft were to fly the trajectory significantly faster, it would fail to meet the state of charge constraint at the end of the trajectory. If left unconstrained, the motor temperature will exceed the $100^{\circ} \mathrm{C}$ limit for about 12 minutes, reaching a peak of $115^{\circ} \mathrm{C}$. Reducing the rate of climb from the $3.81 \mathrm{~m} / \mathrm{s}$ limit to about $2.5 \mathrm{~m} / \mathrm{s}$ is sufficient to prevent violation of the motor temperature limit. The reduction in airspeed when the constraint is active is negligible in terms of the overall performance of the trajectory, adding roughly 10 seconds to the 44 minute flight.
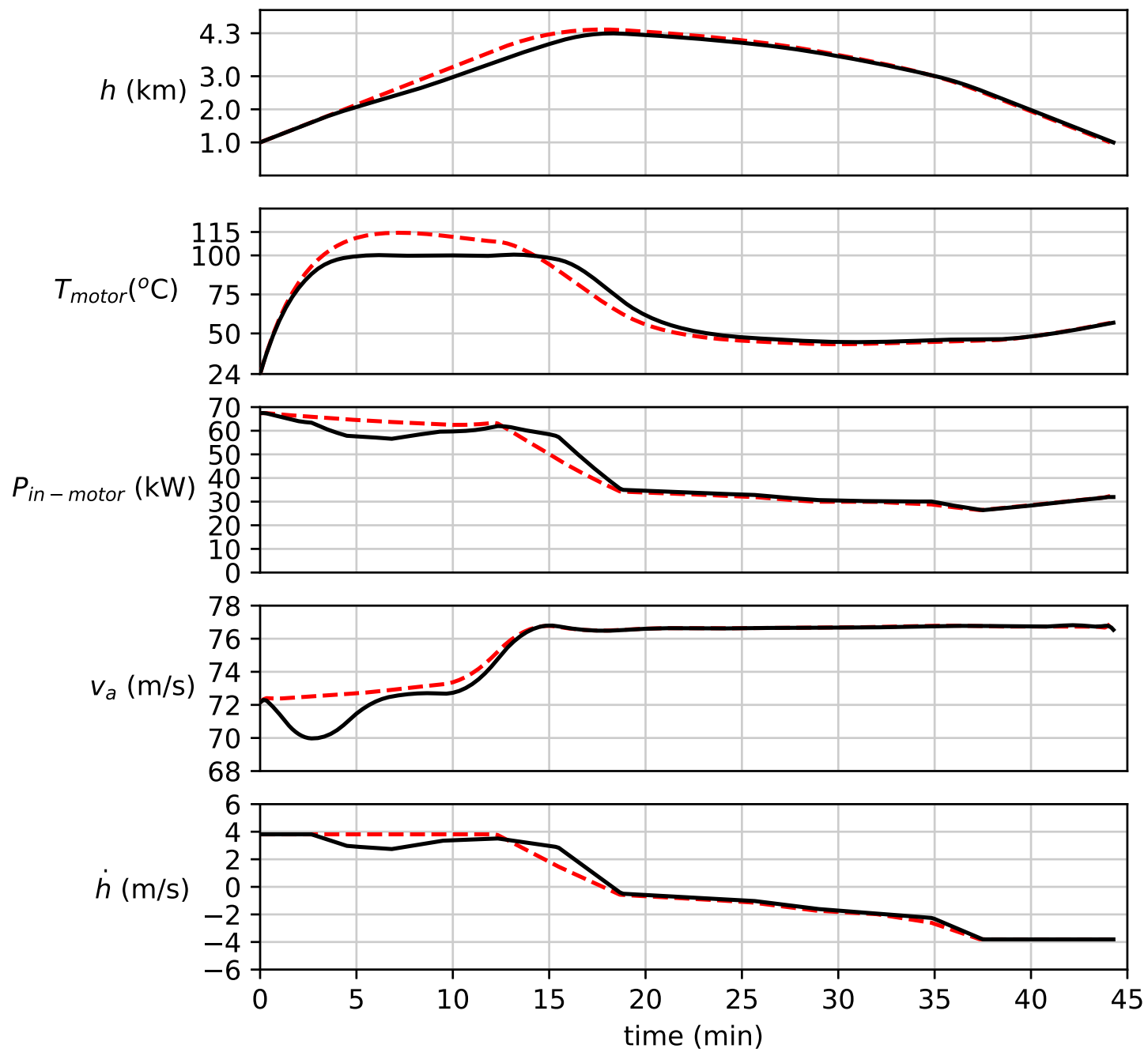

$T_{\text {motor }} \leq 100^{\circ} \mathrm{C} \quad---T_{\text {motor }}$ unconstrained

Figure 9. Comparison of the flight profile and motor temperature for the minimum time case with and without constraints on motor temperature. The temperature-constrained case has a reduced climb-rate before following the nominal trajectory.

\section{Conclusions}

We have demonstrated the ability to conduct trajectory analysis and optimization of electrically powered aircraft, including tracking temperatures of primary electrical components, using a collocation-based 
approach to optimization developed within the OpenMDAO framework. Analysis for this work includes the development of an aircraft model based on the X-57 design. Optimizing the trajectory for maximum range or maximum efficiency shows that the heat rejection systems of the aircraft are adequately sized.

By optimizing the trajectory for maximum range while reducing the cruise motor heat exchanger area by $30 \%$, we demonstrated that the maximum range is reduced less than one half of one percent. In contrast to the range dependence on heat exchanger area, the maximum range is strongly influenced by the limit to the final state of charge of the battery (or the total extractable energy from the battery). With a reduction in heat exchanger area, we found that the motor temperature reaches its maximum allowable value and the aircraft takes an alternate longer duration trajectory with a reduced climb rate that requires less motor input power.

We then developed a minimum time trajectory subject to a fixed range of flight and a limited final state of charge of the aircraft batteries. Using this case, we demonstrated that the thermal load is driven by the initial climb rate, but we can avoid overheating the motor by making a relatively small reduction in the rate-of-climb. If necessary, speed can be increased later during the cruise phase after the motor temperature is reduced with a negligible cost in performance. As in the maximum range case, a requirement that the aircraft complete the trajectory with at least $40 \%$ of its battery capacity proved to be the driving constraint.

In future studies it might be worthwhile to include trajectory optimization in the sizing of the aircraft's heat rejection systems. Given that we can further reduce the thermal load with only minor adjustments to the trajectory (primarily the climb rate), including the sizing of heat rejection systems as independent, static parameters of the optimization would likely result in smaller, lighter heat rejection systems while still meeting all mission requirements. Furthermore, since motor temperature is tightly coupled to motor input power, using a combination of heat rejection capability and trajectory shaping to limit motor temperature would allow for smaller, lower mass motors to accomplish the same missions.

\section{Acknowledgements}

The authors would like to thank Kurt Papathakis and Sean Clarke of NASA Armstrong Flight Research Center for their cooperation and assistance in modeling of the X-57 Maxwell.

\section{References}

${ }^{1}$ Aktas, D., "Electric-Powered Commercial Aircraft Feasibility," 51st AIAA/SAE/ASEE Joint Propulsion Conference, No. AIAA 2015-3889 in Propulsion and Energy Forum, American Institute of Aeronautics and Astronautics, July 2015.

${ }^{2}$ Stoll, A. M. and Mikic, G. V., "Design Studies of Thin-Haul Commuter Aircraft with Distributed Electric Propulsion," 16th AIAA Aviation Technology, Integration, and Operations Conference, AIAA Aviation, American Institute of Aeronautics and Astronautics, June 2016.

${ }^{3}$ Borer, N. K., Patterson, M. D., Viken, J. K., Moore, M. D., Bevirt, J., Stoll, A. M., and Gibson, A. R., "Design and Performance of the NASA SCEPTOR Distributed Electric Propulsion Flight Demonstrator," 16th AIAA Aviation Technology, Integration, and Operations Conference, No. AIAA 2016-3920 in AIAA Aviation, American Institute of Aeronautics and Astronautics, June 2016.

${ }^{4}$ Doman, D. B., "Fuel Flow Control for Extending Aircraft Thermal Endurance Part II: Closed Loop Control," AIAA Guidance, Navigation, and Control Conference, No. AIAA 2016-1622 in AIAA SciTech, American Institute of Aeronautics and Astronautics, January 2016.

${ }^{5}$ Alyanak, E. J. and Allison, D. L., "Fuel Thermal Management System Considerations in the Aircraft Conceptual Design Process," , No. AIAA 2016-0670, 2016.

${ }^{6}$ Vegh, J. M., Alonso, J. J., Orra, T. H., and da Silva, C. I., "Flight Path and Wing Optimization of Lithium-Air Battery Powered Passenger Aircraft," 53rd AIAA Aerospace Sciences Meeting, No. AIAA 2015-1674 in AIAA SciTech, American Institute of Aeronautics and Astronautics, January 2015.

${ }^{7}$ Antcliff, K. R., Guynn, M. D., Marien, T., Wells, D. P., Schneider, S. J., and Tong, M. J., "Mission Analysis and Aircraft Sizing of a Hybrid-Electric Regional Aircraft," 54th AIAA Aerospace Sciences Meeting, No. AIAA 2016-1028 in AIAA SciTech, American Institute of Aeronautics and Astronautics, January 2016.

${ }^{8}$ Christie, R., Dubois, A., and Derlaga, J., "Cooling of Electric Motors Used for Propulsion on SCEPTOR," AIAA Aviation, American Institute or Aeronautics and Astronautics, June 2016.

${ }^{9}$ Dubois, A., van der Geest, M., Bevirt, J., Christie, R., Borer, N. K., and Clarke, S. C., "Design of an Electric Propulsion System for SCEPTOR's Outboard Nacelle," 16th AIAA Aviation Technology, Integration, and Operations Conference, No. AIAA 2016-3925 in AIAA Aviation, American Institute of Aeronautics and Astronautics, June 2016.

${ }^{10}$ Herman, A. L. and Conway, B. A., "Direct optimization using collocation based on high-order Gauss-Lobatto quadrature rules," Journal of Guidance, Control, and Dynamics, Vol. 19, No. 3, 1996, pp. 592-599.

${ }^{11}$ Hargraves, C. R. and Paris, S. W., "Direct trajectory optimization using nonlinear programming and collocation," Journal of Guidance, Control, and Dynamics, Vol. 10, No. 4, 1987, pp. 338-342. 
${ }^{12}$ Gray, J., Moore, K., and Naylor, B., "OpenMDAO: An open source framework for multidisciplinary analysis and optimization," 13th AIAA/ISSMO Multidisciplinary Analysis Optimization Conference, No. AIAA 2010-9101, 2010 , p. 9101.

${ }^{13}$ Falck, R., Gray, J., and Naylor, B., "Parallel aircraft trajectory optimization with analytic derivatives," $17 t h$ AIAA/ISSMO Multidisciplinary Analysis and Optimization Conference, No. AIAA 2016-3207, 2016.

${ }^{14}$ Martins, J. R. R. A. and Hwang, J. T., "Review and Unification of Methods for Computing Derivatives of Multidisciplinary Computational Models," AIAA Journal, Vol. 51, No. 11, 2013, pp. 2582-2599.

${ }^{15}$ Kao, J., Hwang, J., Martins, J., Gray, J. S., and Moore, K. T., A modular adjoint approach to aircraft mission analysis and optimization, No. AIAA 2015-0136, American Institute of Aeronautics and Astronautics, 2016/04/13 2015.

${ }^{16}$ Lambe, A. B. and Martins, J. R. R. A., "Extensions to the Design Structure Matrix for the Description of Multidisciplinary Design, Analysis, and Optimization Processes," Structural and Multidisciplinary Optimization, Vol. 46, 2012, pp. 273-284.

${ }^{17}$ Huria, T., Ceraolo, M., Gazzarri, J., and Jackey, R., "High Fidelity Electrical Model with Thermal Dependence for Characterization and Simulation of High Power Lithium Battery Cells," Electric Vehicle Conference (IEVC), pp 1-8, IEEE International, March 2012.

${ }^{18} \mathrm{He}, \mathrm{H} .$, Rui, X., and Jinxin, F., "Evaluation of lithium-ion battery equivalent circuit models for state of charge estimation by an experimental approach," Electric Vehicle Conference (IEVC), 4.4 582-598, Energies, March 2011.

${ }^{19}$ Gill, P. E., Murray, W., and Saunders, M. A., "SNOPT: An SQP algorithm for large-scale constrained optimization," SIAM Rev., Vol. 47, 2005, pp. 99-131.

${ }^{20}$ Jones, E., Oliphant, T., Peterson, P., et al., "SciPy: Open source scientific tools for Python," 2001-. 